\title{
An Evaluation of Management Placements for nursing students in UK General Practice
}

\section{Authors:}

Nicki Walsh, Programme Lead for Health \& Social Care, Bishop Grosseteste University, Lincoln

Rachael Mason, Practice Placement Development Worker, University of Lincoln, Lincoln

\begin{abstract}
Introduction: General practice is facing a shortage of nurses and it can be difficult to recruit and retain staff. Nursing students need exposure to primary care nursing roles if they are to consider them in their future career and to develop the skills needed for this environment. A study was designed explore the experiences and perceptions of students on their final placement in general practice. The data were thematically analysed and three themes were derived from the data: myth busting, the teaching and learning environment, and attaining competency.
\end{abstract}

Keywords: Assessment, Clinical Placements, Community, Course Evaluation, Education, General Practice, Interprofessional Learning, Mentors, Post-registration Education, Practice Learning, Pre-Registration Education, Primary Care, Sign-Off Mentors, Students, Training, Universities

\section{Introduction}

The UK is rapidly ageing - those older than 65 (the traditional age of retirement) have seen numbers increase by $3.8 \%$ of the population in the last 20 years, with a projected 6.6\% increase by 2039 (Office for National Statistics,2016). Living longer implies that people are more likely to develop long-term conditions (LTCs) such as diabetes, cardio-vascular disease and chronic lung conditions. These people are therefore more likely to experience comorbidities and poor health because of the "natural" ageing process, adding to condition complexity. This makes management more convoluted. Ageing and subsequent frailty can also result in complex social needs putting additional pressures on current services that are already over stretched and under resourced (Kings Fund, 2017). This has significant consequences for all health and social care services, which need to respond dynamically, flexibly, and sustainably to provide safe, effective care while meeting increasing expectations.

"Moving healthcare closer to home" is seen as a way to address some of these issues, with services focusing on providing care in the community through avoiding hospital admissions, effective and quick discharge of patients, smarter use of existing community-based services such as General Practice, and effective use of technology such as telehealth (NHS England, 2015). The movement from secondary care (hospital based), consultant led services, to primary care (primarily GP-led), has 
increased the importance of general practice nurses (GPNs), who now provide most care, especially for long-term conditions (Griffin, 1998; Wells and De Costa, 2005; NHS England, 2015; Simmons et al, 2015).

However, the complexity and the frailty of many of those requiring care requires excellent initial education and training, as well as sustained and consistent informal and formal updates. There must also be a dedication to research to ensure good practice is implemented and effective practice evaluated. This research and education needs to concentrate on what matters to patients, as well as balancing strategic priorities and objectives in services, which are financially under resourced and find it difficult to recruit and retain staff.

NHS England (2016) cited British General Practice as "an expert medical specialist model" that affords excellent, holistic, patient-centered care, as well as a continuous relationship across time which is unique in today's NHS. This requires general practices to tailor treatment to the patient's needs and to act preventively while reflecting on diverse and unique healthcare environments and populations they serve, using staff who are highly skilled and motivated. The right knowledge, skills and attributes of all staff working in General Practice must be attained both foundationally and maintained through effective, quality assured, education and training (Walsh et al, 2011)

\section{Background}

A 1993 survey found that one fifth of nurses working in primary healthcare were GPN's (Atkin et al, 1993). Other roles included district or community nurses, school nurses, and health visitors who are not part of the study discussed in this article because of the differences in their clinical roles and the contributions of their employers to their education and learning. There were few further workforce population surveys, even following the introduction of the quality and outcomes framework in 2004 (British Medical Association, 2003) until the 2015 QNI review of nursing in general practice (QNI, 2015).

Although this review did not estimate the number of nurses working in General Practice in the UK, it did identify that more than one third of GPNs were due to retire by 2020 (QNI, 2015). This raised further concerns around the adequacy of the future workforce's skillsets, as there was no sustainable plan for workforce enhancement in General Practice (QNI, 2015). The review showed evidence that many GPNs have been in roles for several years with little, if any, formal professional development or accredited training which has the potential for a "knowledge hemorrhage" in which clinical experience is less likely to inform clinical judgments and practice, losing what could be described as intuitive practice (Rovithis and Parissopoulos, 2005) or practice wisdom (Knowles, 1984; Benner, et al, 1999).

NHS England $(2015,2016)$ identified a well trained workforce as being critical to moving healthcare closer to home by developing effective recruitment and retention strategies. NHS England (2017) identified the need to provide more convenient access to care and more personalised care in the community, and to concentrate 
more on prevention and population health, to achieve better outcomes and experience for patients. Its ten-point action plan (Figure 1) is a GPN "development strategy, with an extra minimum £15 million national investment which looks at improving training capacity in general practice, increases in the number of preregistration nurse placements, measures to improve retention of the existing nursing workforce and support for return to work schemes for GPNs" (NHS England, 2016).

The sustainability of the future workforce means interest in the role of the GPN must be 'ignited' through undergraduate placements and existing GPNs must be supported with a postgraduate development programme. This article contends that there is an urgent need to think differently about the workforce, especially the skill mix (Centre for Workforce Intelligence 2012), with undergraduate nurses being welcomed into General Practice to experience the breadth of provision, to encourage them to consider general practice nusing as a viable career option (QNI, 2015). NHS England (2017) contained an explicit clause that focuses on increasing preregistration nursing student placements in General Practice. While this is not a new concept, further development and support in General Practice concerning the needs of students is essential (Walsh, 2017). Beyond this initial exposure, however, there must be a sustainability and retention strategy that includes management placements for final-year students, robust preceptorship programmes for newly qualified nurses and a defined career structure.

Figure 1: In summary: the aims of the 10 point plan (NHS England, 2017):

Ten point action plan

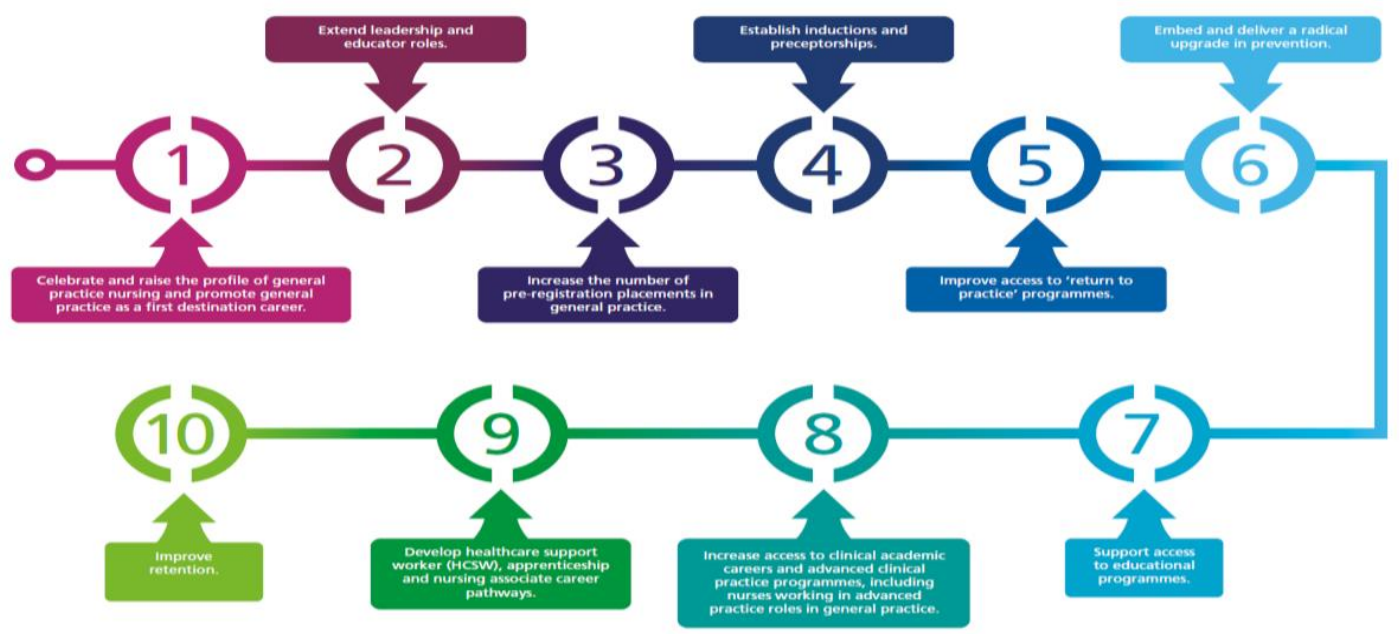

A Health Education England Training Hub initiative intended to develop best practice by engaging and communicating with local clinical commissioning groups (Walsh, 2017). Each Training Hub consists of a designated GP and a nursing lead from Higher Education who recruit GPs to offer education and training to all health professionals, particularly medical and nursing students. The hubs then offer 
external support and encourage internal support within practices around teaching and learning, ensuring an excellent interdisciplinary learning experience. The hubs were rated extremely well by the health professionals, given support and commitment from the Higher Education providers, and provided the opportunity for Inter-professional Education (IPE) that will help shape the future General Practice workforce (Walsh, 2017).

Although GPNs now provide some placements for pre-registration nursing students, the aim of the hubs was to ensure all pre-registration students had a placement in general practice during their undergraduate programme, and those who saw this as a career pathway could undertake their final management placement in General Practice. A previous concern was that students would not be able to attain in general practice the competencies and skills required for entry on the Nursing and Midwifery Council (NMC) register. There was also concern that the autonomy required within General Practice would mean newly qualified nurses would not be able to become GPNs due to their lack of exposure - a somewhat catch-22 situation.

The "Hitting the Ground Running" initiative was developed collaboratively with the Lincoln West training hub and general practices that felt they could support a final placement student. The name was suggested by a GP who supported the initiative as a way of building sustainability and said there was a need for GPNs who could start work with little additional supportive education, except where there were skills needed beyond the scope of their practice.

The aim of the initiative was to develop final management placements in General Practice and evaluate the effectiveness in helping students to enrol on the NMC register.

Objectives were to:

- Enable pre-registration nursing students to have their final placement in general practice to gain skills relevant to becoming registered nurses and the foundation skills needed for general practice.

- Support mentors in GP surgeries to gain the skills to become "sign off mentors" (NMC, 2010) and to offer final placements within their surgeries.

- Evaluate the usefulness of this project from the perspective of students in terms of attaining their final competencies and the value of the experience.

\section{Method}

All final year pre-registration nursing students on one undergraduate programme at a higher education institute (approx. 220) were given a list of placements that had confirmed they could accommodate them for their final placements. This included three general practice settings as well as the more traditional ward and community nursing settings. Students were then asked to complete an application form and provide a CV and supporting statement outlining why they would like a placement in their chosen speciality - this was felt to resemble how they would find future employment as nurses. 
One of the three genera practice settings received several applications and it was decided to make allocation of the placements competitive, as it would be for employment. The GPNs who would provide mentorship were invited to review the applications and decide which students they would support.

All practices underwent a quality assurance audit before offering placements (NMC, 2010). The Higher Education Institute updated and supported the mentors for the final placement by looking at effective teaching and learning in their practices and mapping activity against the NMC competencies to explore how to meet these. Sessions were held for the mentors on what was expected of them during their own development as "sign off mentors".

Meetings with mentors and students were set up at the higher education institute to discuss practicalities and the implications for practice, as well as to look at how they might achieve competencies. Discussion of the students' learning needs was encouraged and a group discussion involving all students and mentors looked at generic concerns. A mapping document was then developed for mentors and students to provide a clear vision of practice opportunities. The participants found the document to be extremely useful, although it needed ongoing reviews and updates.

A further group meeting was held for the intermediate placement interview. The group felt this helped further the discussion of attaining competencies and gave clinicians protected time out of practice to reflect on the students' practice and their own value as enablers of learning.

After the second meeting, one student and mentor expressed their concerns about achieving the placement outcomes defined by NMC (2010). This was a consequence of a discussion the student had had with another academic who wanted to explore how the competencies were being met. The academic undertook a placement visit to explore the competency requirements and aligned them to placement opportunities. Consideration was given to using scenarios and insight visits to enable the student to gain further experience in different practice situations. This particular placement did limit the student's opportunity to assess acutely ill patients. The student and mentor were grateful at the end of the placement visit and felt supported and reassured about meeting competencies.

At the end of the 12-week placement, a member of academic staff (already a 'signoff' mentor) was required to sign off each mentor and student as having successfully attained their competencies. This required the sign-off mentor to visit one placement surgery to sign off a student, as the student's mentor was unable to attend the group signoff, which took place at the higher education institute. After the formal sign-off which was a celebration for all - a group interview was arranged to evaluate the students' experiences.

\section{Methodology}


A group interview with the nursing students who completed their final placement in general practice $(n=3)$ was held to explore their perceptions and experiences of their placement. This obtained qualitative data, deemed the most appropriate method to gain an in-depth view of the student's experiences and perceptions.

\section{Sampling}

The three participants were each given an information sheet outlining the aims of the interview and informed their participation was voluntary. All students decided to participate in the interview and signed consent forms before participation.

\section{Tools}

The group interview was semi-structured and explored how the students had prepared for the placement, the support they received from the higher education institute and their mentors, how they met their competencies, what they felt had been beneficial about their placements, what challenges they had faced, and any suggestions they had for improvements. The authors facilitated the session, which was audio recorded and then transcribed verbatim by an independent transcriber. The students and mentors had already completed an evaluation of practice after the placement as part of the higher education institute's quality assurance, and the interview data were triangulated with evaluation data.

\section{Ethics}

The evaluation was approved by the higher education institute's school ethics committee

\section{Results}

The qualitative data were thematically analysed using Braun and Clarke's (2006) framework and handled on NVivo. The two authors individually analysed the data and then met to reach a consensus on the data's themes. Three main themes emerged from the data:

- Myth busting.

- The teaching and learning environment.

- Attaining competency.

\section{Myth Busting}

Participants reported they had preconceptions about the role of a GPN and completing the placement had changed their views. 
'I thought, oh my God, it is going to be boring'

'...a lot of students have got the view that primary care's boring, which it is really not'

'We (the student nurse and mentor) were talking about the plans of how we were going to meet everything and then I was like, "Oh, I feel better now." I remember saying to her, "We can do this" (participant 2)

'I did not even think you did dressings in a surgery until I went... [I thought] all I am going to be doing is blood pressures.'

'I did not have any background information on what I would be expected to do or what the nurses did in the practice.' (participant 3)

This demonstrated the need for students to be exposed to primary care to understand the roles of GPNs. This may then help to develop this workforce.

There were discussions about the views of nurses and the need to gain experience in a hospital before going into primary care.

'Even the students in our cohort did not realise that they could go and get a job as a practice nurse straight away.'

'I knew you could go into community, which is what I want to do. I do not want to go into a ward, but even when I was on my community placement, they were saying, "Oh, you need to go onto a ward, you need to get all the experience on a ward" (participant 1)

'When I applied to do this degree, I thought that you could just work in a hospital... I even thought you had to do something extra to be a community nurse.' (participant 3)

These comments suggest students and the wider nursing workforce may not be fully aware of the career pathways available to them and further education about different nursing careers may be beneficial.

Two of the participants discussed their experiences before their placements and how these had influenced their choice to return. 
'I would already have been in my placement for my elective, so I should have had four weeks in my placement there. That is why I knew that I wanted to go back.' (participant 1)

'I do think that experience did help me...[go] straight in on my first day and just do things straight away.' (participant 3)

This suggests placements in general practice help to promote the role of the practice nurse and a career in primary care.

\section{Teaching and learning environment}

A prominent theme from the interview was the support participants had from their mentors, the wider GP team and the higher education institute. This included taking time to speak with them, helping them to meet their competencies and providing positive feedback.

'I had three tutors email me to make sure I was okay.'

'Just even asking things that are not particularly to do with student nursing, just where something is kept, or messaging you because there is a message system, "Do you want a cup of coffee?" - it makes you feel part of the team.' (participant 1)

'We get quite a bit of time to sit one-to-one and we have done a lot of teaching. She would block spaces off to teach me and I have learned so much.'

'They are constantly telling you how much you have improved and it is good for your confidence.' (participant 2)

'My mentors have been amazing. All the staff are willing to help. I have had continuous support. They have done everything that I have asked for, in terms of meeting my learning outcomes.'

'I have had an hour's protected time every week.' (participant 3)

These comments demonstrated the value of support from everyone at the placement and the higher education institute in enabling them to complete their placements. The time the mentors provided to the students helped them build their knowledge and skills, as well as to provide feedback, and was invaluable. 
A subtheme concerned their experience of learning while on placement and how this had been positive, especially their relationships with patients. The students also commented on the experiences they had working with other healthcare professionals such as medics, doctors, chiropodists, health visitors, midwives and audiologists.

'It is getting to know the patients and building up that relationship. You get to know them, you get to know when they are not well.'

'I like the age difference. You have got your babies that come in and then you have got your 96 year olds that come in and it is the complete variety.' (participant 1)

'In primary care, you are on your own and you can have that one-to-one time with your patient. I preferred that because you get to build up the relationship better and get to know more about your patient. You have more time. That has been the biggest benefit and improved my consultation skills as well.' (participant 2)

'Following that patient journey, when you are in secondary care, they come in, you treat them, they go and you never know what happens to them or if they are better, whereas in primary care, you can follow that.'

'I say, "I am a final-year nursing student - are you happy to be with me or are you happy for me to do your dressing?" I have not had anybody say no.' (participant 3)

The participants demonstrated that general practice helps their learning and the diverse nature of the patients and their conditions enables them to develop a variety of skills and knowledge.

\section{Attaining competency}

A prominent theme was the development of skills during the placement. These included nursing attributes, such as empathy and improved communication, as well as clinical skills including wound care and knowledge of long-term conditions.

'You have to respect their dignity, let them tell you what they want.'

'Taking bloods, dressings, doing injections, minor surgery, contraception, everything, health reviews.' (participant 1) 
'It makes you feel like a nurse as well, so you are not just an extra pair of hands in a ward. You have actually got your own room and you are seeing your own patients.'

'She [the mentor] did a simulation of a child coming in with an asthma attack and what I would do if that was presented and I was on my own, so that was useful.' (participant 2)

'The history-taking's the important bit in the consultation, isn't it? If you do not get that, then you could be mistreating.'

'The delegation and the management side of it - I said to my mentor, "I do not think I have done that." She replied: "Did you discuss this patient with this nurse and have you referred on?" I said I had: "Me and one of the nurses are sharing the care of a patient." And she said, "Are you going back and updating and things?" I said I was. She said: "You are doing it, but you just do not realise that you are doing it".'

'They might have been having it dressed for four years but I still ask, "How long have you had it?" and "Do you know what started it because you can dress it, but does it need that dressing that is on it now?"' (participant 3)

These comments demonstrate the wide variety of skills students can develop on placements in general practice and how this helps to prepare the student for registration. They can achieve clinical competence while developing relationships based on respect and empathy.

The final subtheme to have arisen from the data was career aspirations based on learning these new skills.

'It is good experience, even for those who do want to go in a hospital, because you could go there and think, "Right, no, this is definitely not for me".'

'I have got a job that is at the practice, yes... I can go on and do my practice nurse degree in January' (participant 1)

I have applied for the RAF. Yes, I would like to be a nurse in the RAF now... They said, "Keep saying that your preference is primary care and you'll get there eventually" (participant 2)

'I have not got a job in my placement as fulltime, but they have put me on the bank, so I can work there and then when a job becomes available, I will be the first to know about it... I would drive 40-odd miles to get to the placement and I would do that.' 
'If it is something that you want, no matter how long it takes, as long as you are always working towards it, you will get there and we can change things.' (participant 3)

This highlights the impact on students' career paths of completing their final placements in general practice and the possible benefits to future workforce recruitment in primary care.

\section{Discussion}

It was acknowledged at the beginning of this pilot project that GPNs had little experience of students undergoing their final placements before registration. There was some apprehension about its usefulness in terms of the experiences that could be offered and support for it from the wider primary care team such as GPs.

Students reported they had preconceptions about the role of a GPN and completing a placement in this environment had changed their views, which demonstrated the need to expose students to primary care throughout their training. Two participants had previously undertaken placements in general practice and their final placements had confirmed their desire to return (Walsh 2017), particularly to gain insight into the role of GPNs and because they aspired to careers in general practice. This exposure will help with recruitment and retention (QNI 2015).

A discussion was held about the view of many nurses - including academics and those in primary and secondary care - that you need to gain experience in a hospital post-registration before going into primary care. Participants agreed that they learned to care in a far more holistic way and relied on their own judgement and autonomy to make decisions more in general practice than they did in secondary care. They felt their confidence to do this was further supported by knowing that their mentor or other members of the team were around, even if they were not present during clinical episodes. This further establishes the need to develop placements in general practice not only for nursing students but others, such as those on health and social care programmes, thus promoting the role of the GPN and focusing on careers in primary care.

A further prominent theme was the support the participants had from their mentors, the wider general practice team and the higher education institute's practice support team. The students felt it was important to take time to understand their learning needs and align these to learning opportunities. Clear action plans of learning were essential to support students and mentors. The mapping document helped to create learning opportunities and could be developed by local surgeries to identify any specific learning required, and any gaps needing further development, such as opportunities to understand the team approach in general practice and valuing everyone involved and their contributions to effective patient care. 
The students said that the time the mentors gave to help them build their knowledge and skills was invaluable and that general practice enabled this due to the nature of the work. They acknowledged that continuous feedback on practice builds practice and professional confidence.

Participants not only identified the team relationships as essential to effective learning but also felt that their relationships with patients were further enhanced by the personalised nature of the roles they performed. Participants alluded to general practice being particularly helpful for their learning and that the diverse nature of the patients and their conditions enabled them to develop a variety of skills and knowledge, whereas certain secondary care environments with specialist focuses would limit their opportunities.

A noticeable theme was the development of skills during the placement. This included nursing attributes, such as empathy, improved communication and providing holistic care, as well as clinical skills such as wound care, venepuncture and management of long term conditions. This demonstrates that a wide variety of skills aligned with NMC requirements are available in a placement in general practice and that through contextual differences, there is greater opportunity to practise these in preparation for registration.

Participants felt that the experiences they gained had not only given them the confidence to work in general practice but offered them a broader practice experience that considered patients' experiences and holistic care requirements that they might not have otherwise experienced. They believed this provided them with a unique skill set that would enable them to become effective practitioners regardless of the settings in which they practised

\section{Limitations}

This article presents an exploration of the perceptions and experiences of three nursing students. The small sample size means the results are not generalisable, but the insight gained is transferable and can be used to inform and enhance practice. When other students undertake their final placements in general practice they may not have the same experiences, so ongoing evaluation would be beneficial to determine if the themes are consistently found and reduce the bias in these results.

The three GPNs that offered final placements to the students were motivated and prepared to commit to this project. Their motivation may have influenced these results, as the support they offered the students may have been due to their interest in the project and their own development as sign-off mentors. Future evaluations of student experiences may help to explore the support offered once placements are more established. 
This evaluation only sought the students' experiences and perceptions. The mentors may have been able to provide further insight and different views of the placements. Future research may wish to explore the experiences of mentors.

\section{Conclusion}

The diverse nature of the general practice nurse's role creates a suitable final placement for nursing students. The support available in general practice provides a positive learning experience for students. Providing nursing students with a final placement in general practice not only helps equip the future workforce with the necessary skills for primary care, it enhances the current workforce's knowledge and skills in terms of their revalidation requirements (NMC 2015). It enables students to consider the possible career pathways open to them in primary care, which serves to support the current workforce and help with the crisis in recruitment and retention.

There are, however, issues that need to be addressed, such as the development of robust preceptorship with peer support and opportunities for peer reflection. There is also the need to review current skill mixes in general practice, to enable new registrants to move into roles that support and promote their development. The GPN ten-point action plan will go some way to support this, but there must be engagement and support in individual practices, from the nursing workforce and practice managers and GPs

\section{Acknowledgements}

The authors would like to thank the mentors that enabled this project to be conducted and for their continued support with student nurse education and the students who participated.

\section{References}

Atkin, K., Lunt, N., Parker, G. and Hirst, M. (1993) Nurses count: a national census of practice nurses. York: social policy research unit. University of York

Braun V, and Clarke V (2006) Using thematic analysis in psychology. Qualitative Research in Psychology. 3, 2, 77-101.

British Medical Association (2003) New General Medical Services Contract. Investing in General Practice. BMA, London.

Benner, P., Hooper-Kyriakidis, P. and Stannard, D. (1999) Clinical Wisdom and interventions in critical care: A thinking- in-action approach. Philadelphia. Saunders 
Centre for Workforce Intelligence. (2012) Shape of the medical workforce: starting the debate on the future consultant workforce. Centre for Workforce Intelligence, [Online]. Available from: http://www.cfwi.org.uk/publications/leaders-report-shapeofthe-medical-workforce

Kings Fund. (2016), Workforce Planning in the NHS, Kings Fund

Kings Fund. (2017) What are the Priorities for Health and Social Care? Accessed on line: https://www.kingsfund.org.uk/publications/what-are-priorities-health-and-social$\underline{\text { care }}$

Knowles, M. (1984). The Adult Learner: A Neglected Species (3rd Ed.). Houston, TX: Gulf Publishing.

Monitor. (2015) Moving care closure to home, Monitor, Gov.co.uk Accessed 28/10/2016

NHS England. (2015) Five-year forward view.

NHS England. (2015) General Practice Forward View: Workforce Plan; https://www.england.nhs.uk/commissioning/primary-care-comm/gp-workforce/

Nursing and Midwifery Council. (2010) Standards of preregistration nursing education. NMC: London

Nursing and Midwifery Council. (2015) Revalidation. NMC: London

Office for National Statistics. (2016) Overview of the UK Population; February 2016; Accessed online; $13^{\text {th }}$ October 2016)

http://www.ons.gov.uk/peoplepopulationandcommunity/populationandmigration/popul ationestimates/articles/overviewoftheukpopulation/february2016

Queen's Nursing Institute. (2015) General Practice Nursing for the $21^{\text {st }}$ Century: a time of opportunity, QNI

Rovithis, M. and Parissopoulos, S. (2005) Intuition in Nursing Practice. ICUS Nursing Web Journal. Issue 22 Available at:

https://www.researchgate.net/profile/Stelios Parissopoulos2/publication/253650486 Intuition in Nursing Practice/links/00b4951fa497cd2298000000/Intuition-in-NursingPractice.pdf?origin=publication detail

Simmons D, Deakin, T, Walsh, $\mathrm{N}$ et al (2015) Diabetes UK Position Statement Competency frameworks in diabetes. Diabetic Medicine. 32, 5. onlinelibrary.wiley.com/doi/10.1111/dme.12702/epdf (Last accessed: 26 September 2018.)

Walsh, N. (2008) Community Nursing: An "Essential" Pre-Registration Nursing Experience - Primary Health Care. Vol 18. No 6, pp18-19 
Walsh N, George S, Priest L et al (2011) The current status of Diabetes Professional Educational Standards and Competencies in the UK - A Position Statement from the Diabetes UK HCP Education Competency Framework Task and Finish Group.

Diabetic Medicine. 28, 12, 1501-1506

Walsh, N. (2017) Using community education provider networks to develop general practice nursing. Primary Health Care, 27, 4, 26-29.

Wells A, and De Costa S (2005) Managed care as a concept meeting the challenge. Journal of Diabetes Nursing. 9, 3. 